\title{
Micro-viscometer based on electrowetting on dielectric
}

\author{
Yan-You Lin ${ }^{\mathrm{a}}$, Chii-Wann Lin ${ }^{\mathrm{a}, \mathrm{b}, *}$, Lung-Jieh Yang ${ }^{\mathrm{c}}$, An-Bang Wang ${ }^{\mathrm{d}}$ \\ ${ }^{a}$ Department of Electrical Engineering, National Taiwan University, Taiwan, ROC \\ ${ }^{\mathrm{b}}$ Institute of Bio-Medical Engineering, National Taiwan University, Taiwan, ROC \\ ${ }^{\mathrm{c}}$ Department of Mechanical and Electro-Mechanical Engineering, \\ Tamkang University, Taiwan, ROC \\ ${ }^{\mathrm{d}}$ Institute of Applied Mechanism, National Taiwan University, Taiwan, ROC
}

Received 16 June 2006; received in revised form 22 September 2006; accepted 11 October 2006

Available online 13 November 2006

\begin{abstract}
A viscometer used to measure the viscosity of $10 \mu \mathrm{l}$ of a liquid, must be miniaturized down, and the liquid velocity gradient in the channel used to determine the viscosity coefficient. Two major factors that affect the liquid velocity are the mechanical forces exerted by the mechanical motors and electromagnetic forces. In this study, electrowetting on dielectric (EWOD) is adopted to drive liquids. Variously sized electrodes on a chip, and two shapes of channel are employed to measure the velocity gradient to determine the viscosity coefficient. The device is fabricated

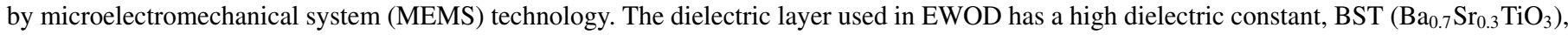
to reduce the required applied voltage; its surface is coated with hydrophobic polymer, polytetrafluoro-ethylene (PTFE, Teflon ${ }^{\circledR}$ AF DuPont). Experimental results demonstrate that liquids can be pulled at $660 \mu \mathrm{m} / \mathrm{s}$ in linear channels by applying a voltage of $15 \mathrm{~V}$.
\end{abstract}

(C) 2006 Elsevier Ltd. All rights reserved.

Keywords: Viscometer; Electrowetting (EW); Electrowetting on dielectric (EWOD); Micro electromechanic system (MEMS); Microfluid

\section{Introduction}

In humans, water in such fluids as interstitial fluid, blood, mucus and others, represents $70 \%$ of the weight. Like their chemical functions, viscosity coefficients are important physical characteristics of the fluids in human bodies. Artificial liquids used as substitutes in living bodies have been examined. In designing a substitute for the aqueous humors in the eyes of human beings, the viscosity coefficient must be determined but only a little of the sample is available in the body. Accordingly, the viscometer must be miniaturized. In this work, the liquid velocity gradient in the channel is employed to find the viscosity coefficient. Two major schemes adopted to drive the liquid involve mechanical forces exerted by motors [1] and electromagnetic forces in the channel. Miniaturizing viscometers and

\footnotetext{
* Corresponding author at: Medical Micro Sensor and System Laboratory, Institute of Bio-Medical Engineering, College of Engineering and College of Medicine, National Taiwan University, No. 1, Sec. 4, Roosevelt Road, Taipei 107, Taiwan, ROC. Tel.: +8862 33665272; fax: +886233665268.

E-mail address: cwlinx@ @tu.edu.tw (C.-W. Lin).
}

reducing the volume of liquid required by EW are of great interest [2].

Since the advancement of MEMS, there have been many devices studied and developed to manipulate liquids in microfluid channels. For all these devices, there are various actuation methods to pump or control fluid, such as piezoelectric, electrostatic, thermopneumatic, electromagnetic, electrophoretic, electroosmotic, etc. [3-5] Due to the process limitation, device reliability and cost of micromachining, the actuation mechanism with the least fabrication complication in devices is absolutely needed for microfluidics. Surface tension, which is always neglected on a macroscale, becomes dominant force on a microscale. Since surface tension cannot be avoid on the microscale, electrowetting (EW) utilizes the surface tension to be wetted a liquid by applying voltages [6-9]. The contact angle will be changed and the surface tension varies, while the applied voltage affects the electric field and electrons distribution in an electric double layer (EDL) for EW or a dielectric layer for electrowetting on dielectric (EWOD) [9]. EWOD has been used for analysis of peptides and proteins [10]. In this study, EWOD is used to drive the flow in the designed channels and produces the velocity gradients to measure viscosity coefficient. 


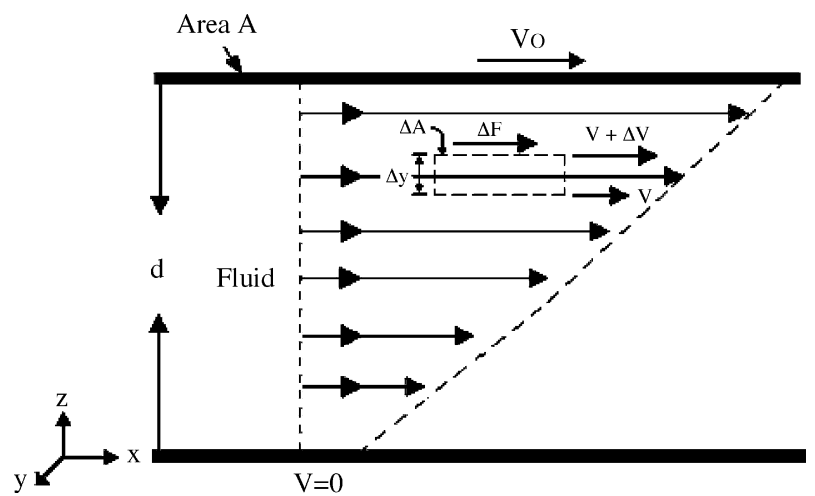

Fig. 1. Flow situation in static and dynamic conditions.

\section{Theory and principle}

\subsection{Viscometer}

When a shear strain is applied to one side of a liquid in a channel to cause it to flow, the viscosity relates these shear forces to the motion of the fluid. At rest, a shear force is applied to the upper side of two parallel plates to induce a flow at a slow speed $v_{0}$, whereas the other plate is held stationary, as presented in Fig. 1 in the top and the bottom of the channel. The shear strain is

$\frac{F}{A}=\eta \frac{v_{0}}{d}$

[11], where the parameter $\eta$ is the viscosity coefficient and $d$ is the thickness of the channel.

For an infinitesimal rectangular area in the flow between two plates, as in the one-dimensional case shown in Fig. 1, the shear stain is given by

$\frac{\Delta F}{\Delta A}=\eta \frac{\Delta v_{x}}{\Delta y}, \quad \lim _{\Delta \rightarrow 0} \eta \frac{\Delta v_{x}}{\Delta y}=\eta \frac{\partial v_{x}}{\partial y}$

In the two-dimensional case, the general form of the shear strain is

$S_{x y}=\eta\left(\frac{\partial v_{y}}{\partial x}+\frac{\partial v_{x}}{\partial y}\right)$

Consider a rotational fluid and the boundary conditions for a point at $y=0$ : as shown in Fig. 2, $\partial \omega / \partial y=0 ; x(\partial \omega / \partial x)=r(\partial \omega / \partial r)$, and the shear strain is

$\left(S_{x y}\right)_{y=0}=\eta r \frac{\partial \omega}{\partial r}$

In a circular channel, every point has $y=0$. For circular motion, $v_{t}=r \omega$, and the shear strain is related to the velocity according to

$S_{\mathrm{cir}}=\eta r\left(\frac{1}{r} \frac{\partial v_{t}}{\partial r}-\frac{v_{t}}{r^{2}}\right)$

Then, the viscosity coefficient is determined by the speed of the liquid at various radii.

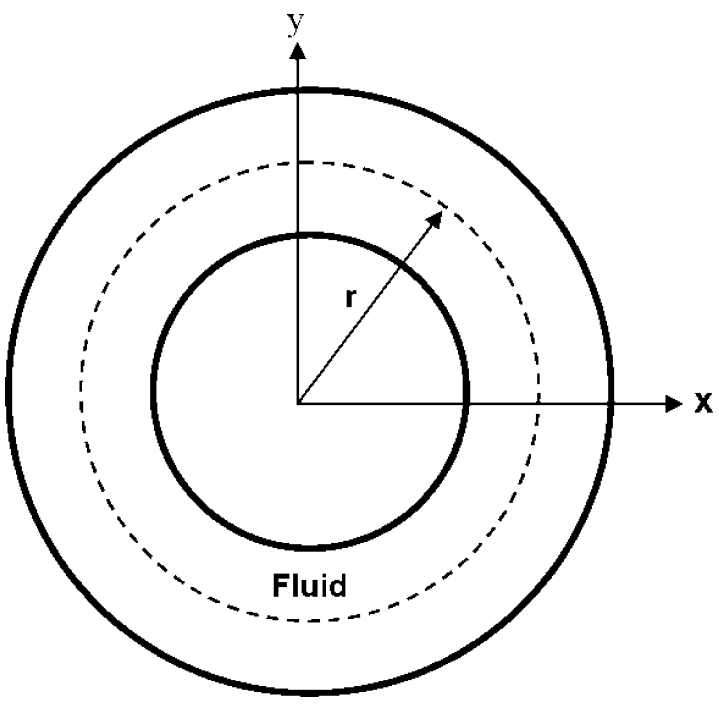

Fig. 2. Fluid flows in a circular channel.

\subsection{Electrowetting}

The relationship between the applied voltage and the surface tension among liquid, electrodes and air can be derived at the interface. The contact angle is a function of the applied voltage between liquid and electrode [9]. As the contact angle varies with the applied voltage, the surface wettability varies between hydrophobic and hydrophilic. Three electrowetting methods are available; they are continuous electrowetting (CEW) [7], electrowetting (EW) $[6,12]$, and electrowetting on dielectric (EWOD) $[6,8]$. EW drives flow in a channel by varying the surface tension, which depends on the electrical double layer (EDL), between the interface of the channel, air and the liquid. In EWOD, the EDL is replaced by the dielectric material $\left(\mathrm{Ba}_{0.7} \mathrm{Sr}_{0.3} \mathrm{TiO}_{3}, \mathrm{BST}\right)$ and a hydrophobic polymer (PTFE, Teflon ${ }^{\circledR}$ AF) [8].

Based on Lippmann-Young's equation [6]:

$\cos \theta=\cos \theta_{0}+\frac{1}{\gamma_{\mathrm{LG}}} \frac{1}{2} c V^{2}$

where $\theta$ is the contact angle, $\theta_{0}$ the contact angle when the electric field across the interfacial layer is zero, $\gamma_{\mathrm{LG}}$ the liquid-gas surface tension, and $c$ is the capacitance per unit area of the EDL or the dielectric layer. The droplet is wetted by the applied voltage in EWOD and remained wetted as the voltage is removed. The droplet becomes hydrophobic again after the applied voltage is grounded. The thickness of the dielectric affects the wettability of the liquid. The pressure induced by the surface tension due to a change in contact angle is given by [6]:

$\Delta p=p_{\mathrm{L}}-p_{\mathrm{R}}=\frac{\gamma_{\mathrm{LG}}}{d}\left(\cos \theta_{\mathrm{b}}-\cos \theta_{\mathrm{b} 0}\right)$

The Lippmann-Young's equation is

$\Delta p=\frac{1}{2 \mathrm{~d}} c V^{2}=\frac{\varepsilon_{0} \varepsilon V^{2}}{2 \mathrm{~d} t}$

where $\varepsilon$ and $t$ are the dielectric constant and the thickness of the surface material, respectively. 
Table 1

The pros and cons of each method [6-9]

\begin{tabular}{|c|c|c|}
\hline CEW & EW & EWOD \\
\hline \multicolumn{3}{|l|}{ Advantages } \\
\hline 1. Low power & 1. Mid power & 1. Repeatable \\
\hline 2. High speed & 2. Easy fabrication & 2. Suit for all polar liquid \\
\hline 3. Smooth operation & & 3. Easy fabrication \\
\hline \multicolumn{3}{|l|}{ Disadvantages } \\
\hline 1. Fabrication is complex & 1. Unrepeatable & 1. High power \\
\hline 2. Need the liquid metal & $\begin{array}{l}\text { 2. Electrodes' } \\
\text { oxidation } \\
\text { 3. Choices of the } \\
\text { electrolyte }\end{array}$ & $\begin{array}{l}\text { 2. Material choice of the } \\
\text { dielectric }\end{array}$ \\
\hline
\end{tabular}

Tables 1 and 2 compare these approaches of each method [6-9].

According to both tables, CEW requires mercury in the channel, and the process becomes complex, potentially damaging the sample. The EW channel is not reusable and the liquid may be electrolyzed due to the conduction of electrodes and liquid. The conclusion is that EWOD is preferred for use in the experiment [13].

\section{Experimental procedure}

Based on theories concerning the viscometer and EW described above, EWOD is utilized to drive the flow on the one side of the channel and the velocity of the liquid is observed on the opposite side. The external force exerted by EWOD is

$F_{\mathrm{ex}}=\Delta p \Delta A_{\mathrm{cross}}=\frac{\varepsilon_{0} \varepsilon V^{2}}{2 \mathrm{~d} t} \Delta A_{\mathrm{cross}}$

where $\Delta A_{\text {cross }}$ represents the cross-sectional area of the channel. In the linear channel, the viscosity coefficient is related to the external force by

$F_{\text {ex }}=S_{1} \Delta A_{\tan }=\eta\left(\frac{\partial v_{x}}{\partial y}+\frac{\partial v_{y}}{\partial x}\right) \Delta A_{\tan }$

where $\Delta A_{\tan }$ is the tangent area that is in contact with the liquid and the channel wall. For a curved channel, which is part of a circular channel, the viscosity coefficient is related to the external force by

$F_{\mathrm{ex}}=S_{\mathrm{c}} \Delta A_{\tan }=\eta r\left(\frac{1}{r} \frac{\partial v_{t}}{\partial r}-\frac{v_{t}}{r^{2}}\right) \Delta A_{\mathrm{tan}}$
Table 3

Design of curvature electrodes and channels

\begin{tabular}{lc}
\hline Channel width $(\mu \mathrm{m})$ & Electrode size with radius \\
\hline 500 & $1000 \mu \mathrm{m}$ with radius $4000 \mu \mathrm{m}$ \\
1000 & $500 \mu \mathrm{m}$ with radius $2500 \mu \mathrm{m}$ \\
2500 & $250 \mu \mathrm{m}$ with radius $1000 \mu \mathrm{m}$ \\
& $100 \mu \mathrm{m}$ with radius $500 \mu \mathrm{m}$ \\
\hline
\end{tabular}

Table 4

Design of straight electrodes and channels

\begin{tabular}{lc}
\hline Channel width $(\mu \mathrm{m})$ & Electrode size with radius $(\mu \mathrm{m})$ \\
\hline 500 & 1000 \\
1000 & 500 \\
2500 & 250 \\
& 100 \\
\hline
\end{tabular}

Numerous physical considerations influence Eq. (11), such as the channel size, the dielectric constant of the material, the thickness of the material and the size of the electrode. Eqs. (9) and (10) reveals that the linear channel viscosity coefficient $\eta_{1}$ for the linear channel is

$\eta_{1}=\frac{1}{\left(\partial v_{x} / \partial y\right)+\left(\partial v_{y} / \partial x\right)} \frac{\varepsilon_{0} \varepsilon V^{2}}{2 \mathrm{~d} t} \frac{\Delta A_{\text {cross }}}{\Delta A_{\tan }}$

Also from Eqs. (9) and (11), the viscosity coefficient $\eta_{\mathrm{c}}$ in the curved channel is

$\eta_{\mathrm{c}}=\frac{1}{\left(\partial v_{t} / \partial r\right)-\left(v_{t} / r\right)} \frac{\varepsilon_{0} \varepsilon V^{2}}{2 \mathrm{~d} t} \frac{\Delta A_{\mathrm{cross}}}{\Delta A_{\mathrm{tan}}}$

Rearranging Eq. (13) yields:

$\left(\frac{\partial v_{t}}{\partial r}-\frac{v_{t}}{r}\right)=\frac{1}{\eta} \frac{\varepsilon_{0} \varepsilon V^{2}}{2 \mathrm{~d} t} \frac{\Delta A_{\mathrm{cross}}}{\Delta A_{\mathrm{tan}}}=\frac{c_{0}}{\eta}$

where the constant $c_{0}$ can be determined by substituting values into Eq. (14). Integrating Eq. (14) yields:

$v_{\mathrm{t}}=r\left(-\frac{c_{0}}{\eta} \ln r-c_{1}\right)$

which is the relationship between the tangential velocity $\left(v_{\mathrm{t}}\right)$, the radius $(r)$, the viscosity coefficient $(\eta)$ and the integral constant $c_{1}$. The vacuum dielectric constant $\varepsilon_{0}$ is $8.85 \times 10^{-12} \mathrm{~F} / \mathrm{m}$; the BST dielectric constant $\varepsilon_{\mathrm{r}}$ is 180 , its thickness $t$ is $80 \mathrm{~nm}$, the channel height $d$ is $50 \mu \mathrm{m}$ and the applied voltage $V$ is $20 \mathrm{~V}$. The electrodes and channels are designed in linear and curvature arrays, as described in Tables 3 and 4. The edges of the square electrodes are designed with isosceles triangles to have

Table 2

Major parameters of each method [6-9]

\begin{tabular}{llll}
\hline & EW & EWOD with Teflon & EWOD with BST \\
\hline Applied voltage $(\mathrm{V})$ & $\sim 1$ & $50-100$ & $\sim 15(15-50)$ \\
Dielectric constant, $\varepsilon$ & 80 & 2 (Teflon) & 180 (BST) \\
Thickness & $100 \AA$ (EDL) & $100 \mathrm{~nm}$ (Teflon) & $100 \mathrm{~nm}($ BST) \\
Channel height & $10 \mu \mathrm{m}$ & $10 \mu \mathrm{m}$ & $10 \mu \mathrm{m}$ \\
Pressure (Pa) & $\sim 3.5 \mathrm{kPa}$ & $\sim 9 \mathrm{kPa}$ & $\sim 180 \mathrm{kPa}$ \\
\hline
\end{tabular}




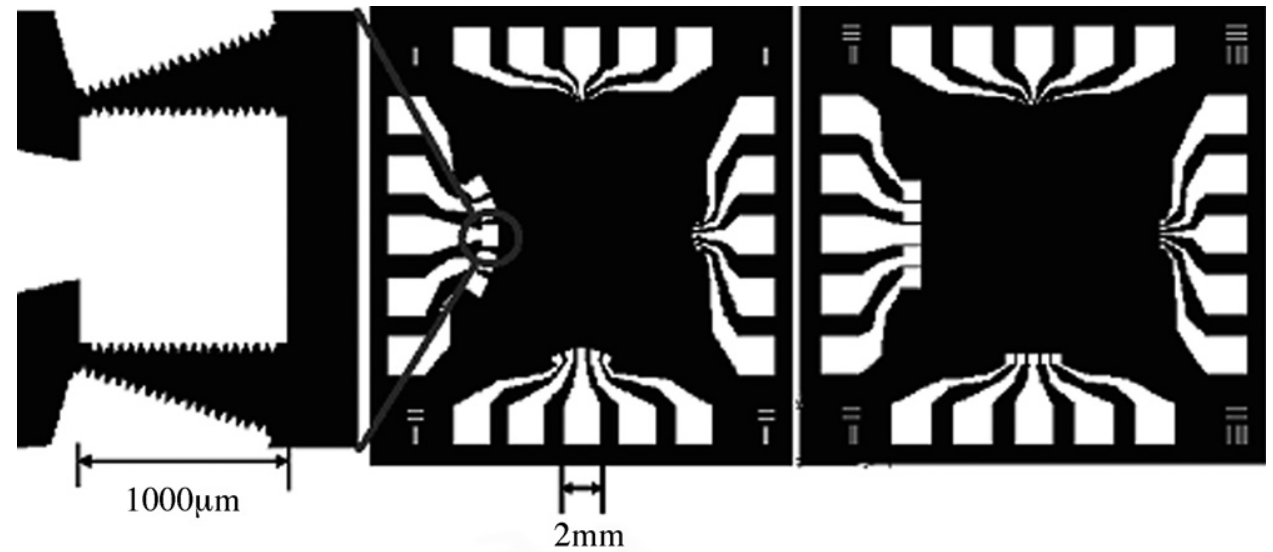

Fig. 3. Layout of two kinds of electrodes.

the lowest potential energy gradient with sides of length $50 \mu \mathrm{m}$, as shown in Fig. 3. The potential energy gradient of the triangles is only higher than sinusoidal wave shape edges, which are difficult exactly defined by photolithography [14].

The fabrication process is as presented in Fig. 4. Glass-based wafers are applied as substrates in the device. After the standard cleaning process, a layer of the photoresist (Negative photoresist, SPR220) is spin coated on the substrate, and the electrodes are defined on wafers by lift-off. The wafers are etched to a depth of $120 \mathrm{~nm}$ by buffered oxide etch (BOE) in the shape of an electrode; $\mathrm{Cr}$ and $\mathrm{Pt}$ are deposited on the device using an E-beam evaporator with thicknesses of 20 and $100 \mathrm{~nm}$, respectively. For EWOD, a dielectric layer is required between the electrodes and the hydrophobic surface layers (PTFE, Teflon ${ }^{\circledR}$ AF) $[15,16]$. Also, according to Eq. (8), a thin material with a high dielectric

(a)

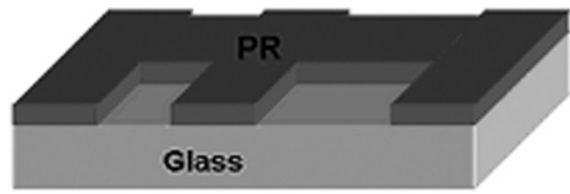

(b)

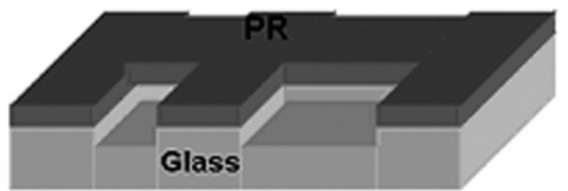

(c)

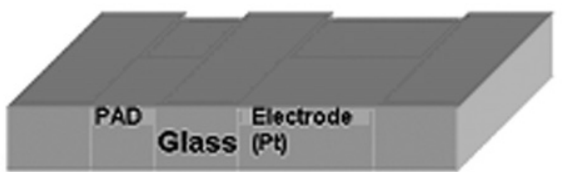

(d)

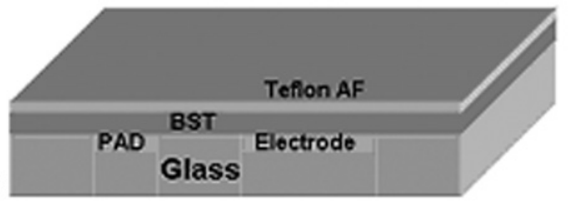

constant is associated with a low required voltage. BST is the preferred choice because of its high dielectric constant. The electrode surfaces were hydrophobic when no voltage was applied, and were hydrophilic ones when a voltage was applied. Teflon ${ }^{\circledR}$ AF was the surface material, which was spin coated to a thickness of $20 \mathrm{~nm}$. The reactive ion etching (RIE) was Teflon ${ }^{\circledR} \mathrm{AF}$ etching, and BOE used for the BST etching. The channel wall was photolithographed using a negative epoxy-based photoresist, SU-8 [17], with a thickness of approximately $40 \mu \mathrm{m}$. The topside was covered by glass coated with indium tin oxide (ITO) and Teflon ${ }^{\circledR}$ AF as grounded.

A LabVIEW program is designed to control the applied voltage in the five electrodes. The program is a one-to-five multiplexer that applies voltages; the duty cycles are tunable using the panel. The power supply applies $15 \mathrm{~V}$ and the time interval

(e)

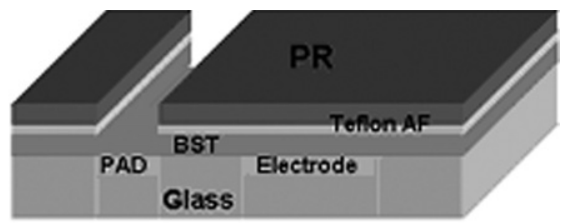

(f)

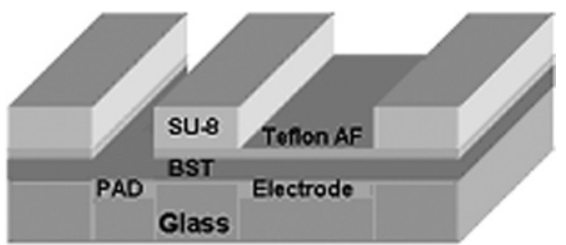

(g)

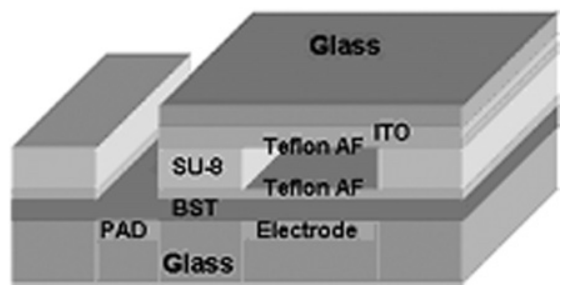

Fig. 4. Device process: (a) the patterns of electrodes are defined on the glass substrate by lift-off with negative photoresist (PR); (b) etch the substrate with BOE for $120 \mathrm{~nm}$; (c) deposit electrodes with $\mathrm{Cr}(20 \mathrm{~nm})$ and Pt $(100 \mathrm{~nm})$ and remove photoresist; (d) deposit BST and coat Teflon ${ }^{\circledR}$ AF; (e) photolithograph pads with photoresist and etch Teflon ${ }^{\circledR}$ AF with RIE and BST with BOE; (f) photolithograph SU-8 as channel wall; (g) bonding with ITO coating Teflon ${ }^{\circledR}$ AF. 


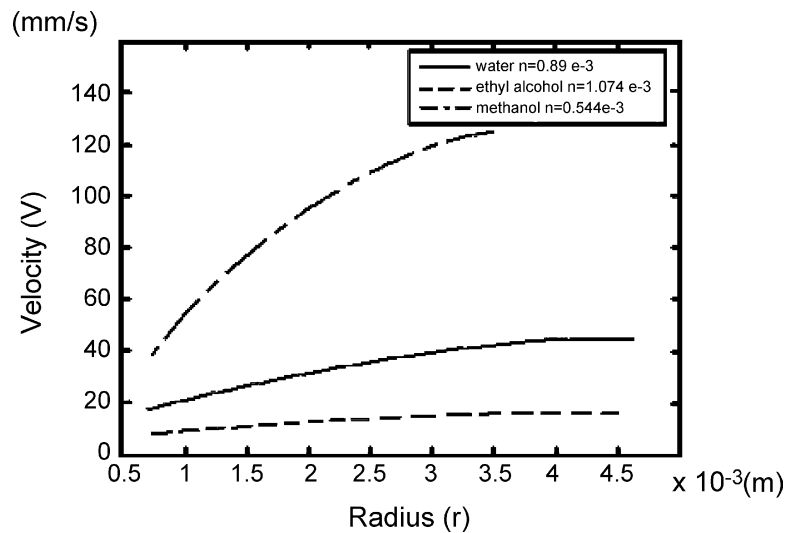

Fig. 5. Simulation of "velocity vs. radius" through water, ethyl alcohol, and methanol. Various liquids have different velocities relating to the radii.

is $100 \mathrm{~ms}$. The time required for liquids to flow through the electrodes and to be displaced at various positions can be obtained from continuous images captured using a CCD camera through a microscope. A polynomial formula is then obtained by fitting the curve from the velocity-position plot, and the viscosity coefficient is estimated using Eqs. (12), (13) and (15).

\section{Results}

\subsection{Simulation results}

The constant $c_{0}$ is evaluated using Eq. (14) by substituting the numerical values of the parameters. The viscosity coeffi-

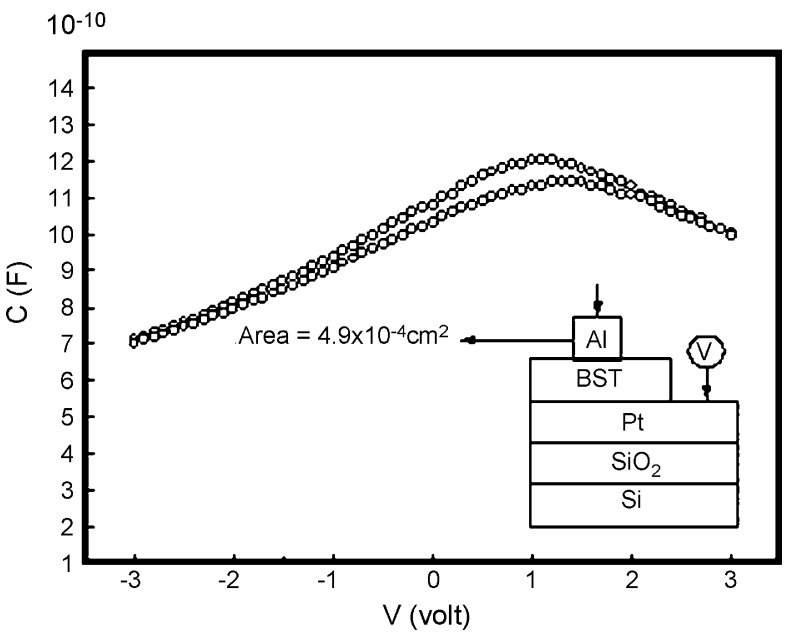

Fig. 6. Measurement of "capacitance vs. voltage of BST" and testing sample chip structure. BST has varied dielectric constants when applying different dc voltage due to its unique properties.

cient curves for three liquids, water ( $\eta$ is $0.89 \times 10^{-3} \mathrm{~Pa} \mathrm{~s}$ ), ethyl alcohol ( $\eta$ is $1.074 \times 10^{-3} \mathrm{Pas}$ ) and methanol $(\eta$ is $0.544 \times 10^{-3} \mathrm{~Pa} \mathrm{~s}$ ), are obtained from the equation. Fig. 5 displays the results, as the velocity ranges from 10 to $150 \mathrm{~mm} / \mathrm{s}$ and the radii vary from 500 to $5000 \mu \mathrm{m}$.

\subsection{Experiment results}

Fig. 6 plots the capacitance of BST, from which the dielectric constant is determined. A BST film layer as thin as $0.1 \mu \mathrm{m}$ and

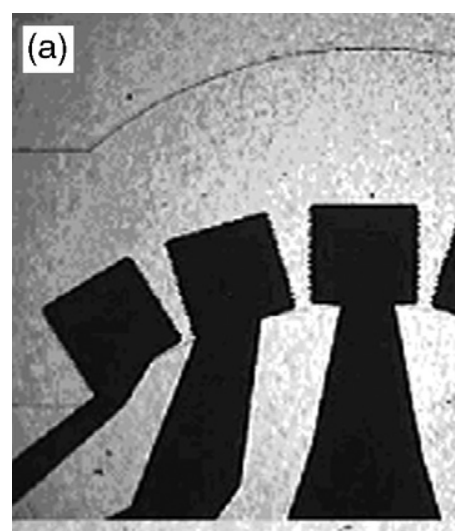

(c) (b)

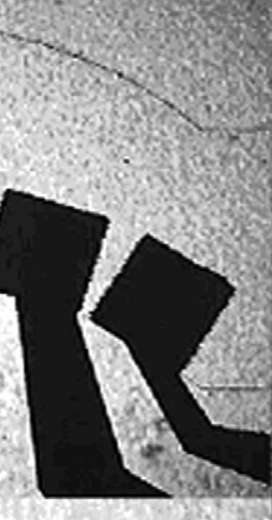

(2) $($ d)

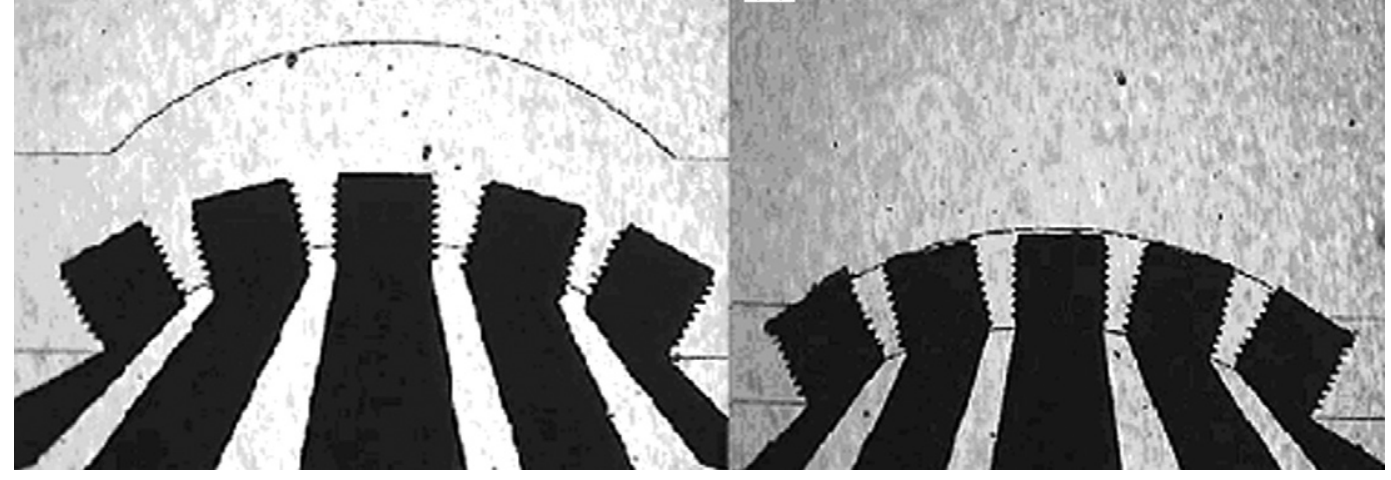

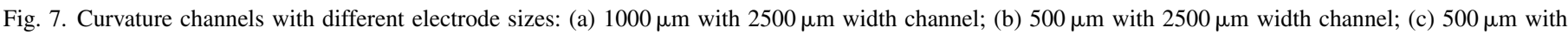
$1000 \mu \mathrm{m}$ width channel; (d) $500 \mu \mathrm{m}$ with $500 \mu \mathrm{m}$ width channel. 


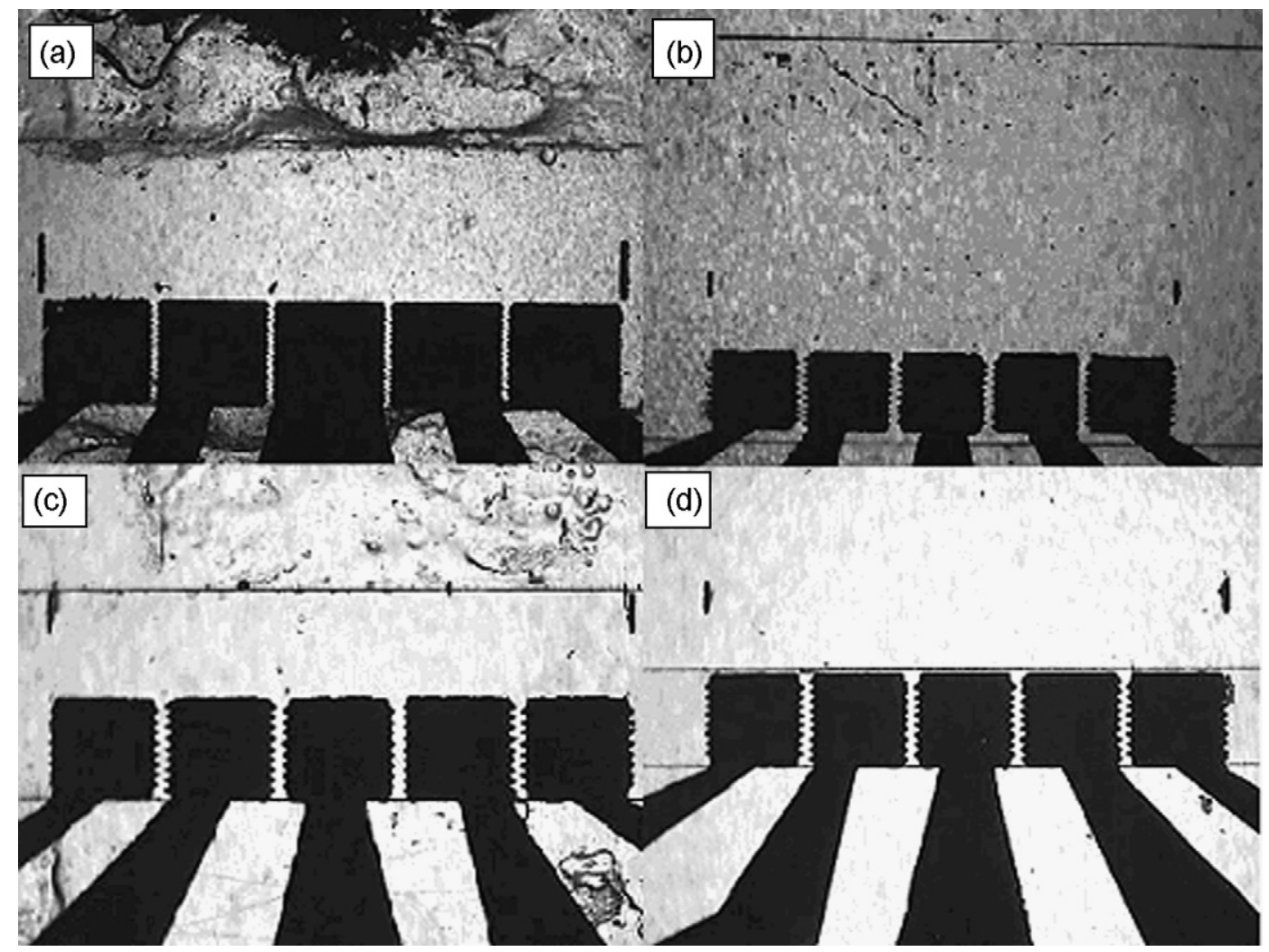

Fig. 8. Linear channels with different electrodes sizes: (a) $1000 \mu \mathrm{m}$ with $2500 \mu \mathrm{m}$ width channel; (b) $500 \mu \mathrm{m}$ with $2500 \mu \mathrm{m}$ width channel; (c) $500 \mu \mathrm{m}$ with $1000 \mu \mathrm{m}$ width channel; (d) $500 \mu \mathrm{m}$ with $500 \mu \mathrm{m}$ width channel.

not uniform over its whole area, electrodes with a small area are fabricated on the testing chip. The dielectric constant is related to the capacitance by

$C=\frac{\varepsilon_{0} \varepsilon A}{t}$

where $\varepsilon_{0}$ is $8.85 \times 10^{-12} \mathrm{~F} / \mathrm{m}$, the electrode area $A$ $4.9 \times 10^{-8} \mathrm{~m}^{2}$, and the thickness $t$ is $80 \mathrm{~nm}$. From the $C-V$ curve in Fig. 6 , the BST capacitance is between $7 \times 10^{-10}$ and $1.2 \times 10^{-9} \mathrm{~F}$ at various voltages. The dielectric constant ranges from 129.13 to 221.37 .

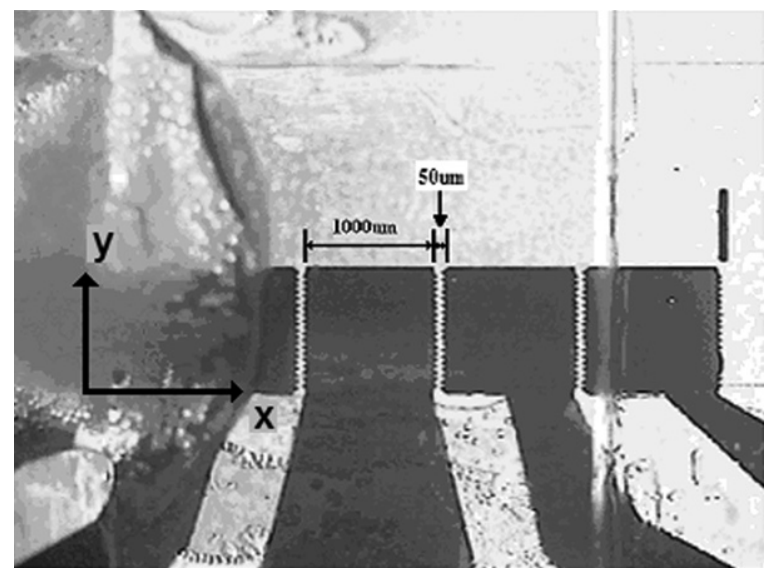

Fig. 9. Liquid flows in $2500 \mu \mathrm{m}$ width channel with $1000 \mu \mathrm{m}$ electrodes and measuring the velocity gradients.
The electrodes and channels are fabricated by the MEMS process. Figs. 7 and 8 show the curvature and linear channels with various widths and variously sized electrodes. Saline $(0.9 \%$ $\mathrm{NaCl}$ solution) solution is used as the sample. A velocity gradient is induced by the flow of liquid in a $2500 \mu \mathrm{m}$-wide linear channel with $1000 \mu \mathrm{m}$ electrodes, as shown in Fig. 9. In a linear system, four positions are selected and the relationships between the velocity and the position in the $x$ - and $y$-directions are as plotted in Figs. 10 and 11, respectively. Then, $\partial v_{x} / \partial y$ and $\partial v_{y} / \partial x$ are evaluated by finding the first-order differentiations to be substi-

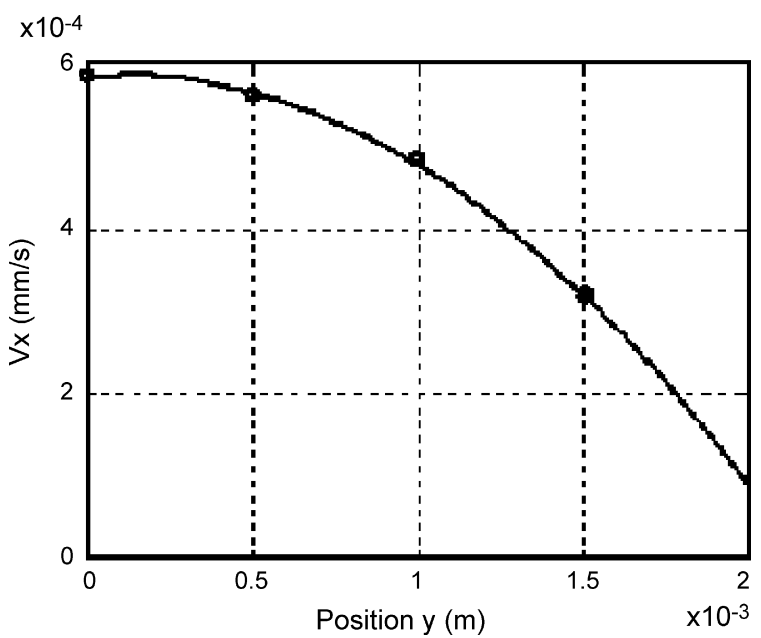

Fig. 10. $V_{x}$ vs. position $y$ curve with second-order curve fitting. The polynomial equation is $V_{x}=0.0005809+0.0378 y-142 y^{2}$. 


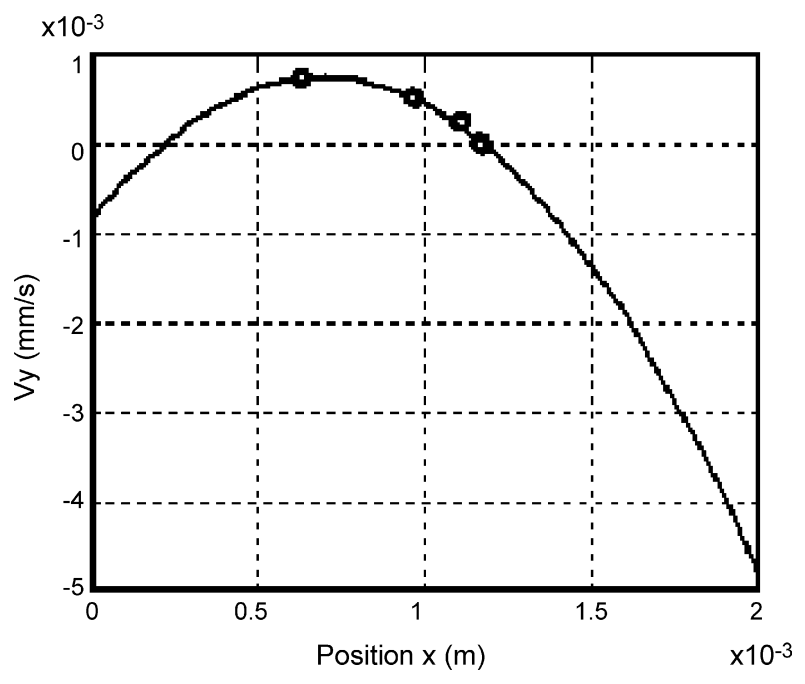

Fig. 11. $V_{y}$ vs. position $x$ curve with second-order curve fitting. The polynomial equation is $V_{y}=0.00081648+4.5281 x-3250.8058 x^{2}$.

tuted into Eq. (12). The viscosity coefficient, which is 94.28 for saline solution, is thus determined.

\section{Discussions}

A micro viscometer based on EWOD can manipulate liquid on a micro scale, and the chip area is $2.3 \mathrm{~cm} \times 2.3 \mathrm{~cm}$. All analysis process is accomplished only in a few seconds. As the advancement of MEMS technology, simple and fast processes by mass production can easily approach the purpose of cost down. However, the device still has disadvantage that EWOD needs higher level of voltage. Even though the applied voltage can be significantly reduced with BST layer, it is still much higher than EW and CEW. The dielectric material and deposition quality is the key point to decrease the applied voltage with well liquid manipulation.

In our experiments, the BST surface is not uniform due to the deposition quality and it results in the thickness variation through whole wafer. While etching BST, the glass below electrodes would be under cut and the electrodes will split out. Additionally, the dielectric constant varies as the thickness is not uniform. Moreover, some thin area is easily punched through and results pin holes, and the liquid touching with the electrode surface directly will electrolyze and produce bubbles to interfere the observation. A great amount of bubbles induce flows in different directions and block the liquid flow. Besides, the electrodes, liquid, and ITO glass will conduct as a circuit, and the liquid can be considered as a resist. When applying voltages, the electrical current flows through the resist and induces heat. The viscosity coefficient is sensitive to temperature. Furthermore, the water in solution vapors rapidly due to the heat, and the sample therefore becomes dense resulting in the variation of the viscosity. As the high frequency voltage is applied, the bubble effects can be improved, but there are still tiny bubbles locally inducing at the edge of electrodes. While the applying voltage interval is shortened, the heat caused by circuit can be reduced. The uniform BST deposition and better process quality can release the pin hole effects and improve problems above.

Except for the material process of EWOD, the bonding efficiency at the ground cover is another issue in the experiment. Since ITO glass served as ground bonds with SU-8 channel wall by UV glue, the glue cannot precisely spread and firmly seal at the sides of channel, and there are some spaces induced along the side. As the result, liquids will overflow obviously from the spaces due to the capillary effects. The capillary effects show not only at the sides of channel, but also the width and length ratio of channel. The wide width and short length channel makes capillary stronger. When the liquid is dropped in channel, it will be pulled to another side immediately. Teflon AF coated at the surface is hydrophobic but after the channel is wetted once, the wall becomes a little more hydrophilic due to the liquid cohesion and the liquid will pass through the channel rapidly. As the reason, after the liquid flows through the channel, we have to ground each electrode to let them recover to hydrophobic state and dry out the channel at the same time. Because of the conductibility and transparency, ITO glass is chosen as a cover and ground in the device. But in the experiment, we find that the ITO at cathode burns out as the voltage applied and loses its conductibility.

In the micro channel, besides capillary effects, the cohesion and surface tension of liquid, and resistance between liquid and channel will show its influence. Before the flow motion, the cohesion and surface tension of liquid outside the channel makes it more difficult to start motion. After the liquid flows, not only bubbles and heat affect the result, but also the resistance consume part of work applied by EWOD, and the velocity cannot reach the speed in ideal simulation.

Finally, the electrodes design in our devices, we assumed the force described by viscosity as a shear strain that acts on a tangent area is equal to the force induced by EWOD as a pressure that suppresses on a sectional area of channel. The assumption is proved while the driving force is at the edge of the channel to be regarded as a shear force. As a result of this condition, the channel width to electrode size ratio ought to be large and can still pull the flow on the opposite side. But in the experiment, the liquids get better control on the 500 and $1000 \mu \mathrm{m}$ electrodes. Under the larger ratio, these two forces may not be regard as equal. The design can be used in the future is the device which electrodes and channel are the same size and the image captured from the side of chip. Furthermore, according to the EWOD theory, while the liquid is on one electrode, the liquid should cover the other electrode beneath and then the surface can be wetted to drive liquids. For the curvature system, because the electrodes are arranged in an arch shape, the gaps between electrodes are too far at the periphery to cover the next electrodes and result in failure.

In our system, due to the high level applied voltage, the high level power supply and the LabVIEW system are still needed and cannot be integrated on chip. If we can reduce the voltage under 3 or $5 \mathrm{~V}$, the control circuits can utilize semiconductor and IC design to integrate into the device. The detection system is optical microscope with image frame grabber, but the light source is another heat source for samples. The micro velocity 
detector which can get more accurate velocity and do not affect the results integrates in micro channels and then the whole system can be scale down.

\section{Conclusion}

This work designed a novel EWOD micro viscometer, a novel EWOD micro viscometer was designed based on theoretical derivations. The liquid velocity gradient in the channel is considered to determine the viscosity coefficient. The simulation results reveal the feasibility of measuring viscosity coefficients of solutions in the range $0.5 \times 10^{-3}$ to $2 \times 10^{-3} \mathrm{~Pa} \mathrm{~s}$, corresponding to velocities from 5 to $140 \mathrm{~mm} / \mathrm{s}$ when $20 \mathrm{~V}$ is applied. The use of EWOD to drive the flow is demonstrated, and a miniaturized viscometer with a simple multiplexer control circuit is realized. Experimental results demonstrate that liquids can be pulled in linear channels by applying $15 \mathrm{~V}$ and a velocity of saline solution $660 \mu \mathrm{m} / \mathrm{s}$ at the electrodes with a resulting viscosity coefficient of 94.283 . The viscometer based on a CCD camera used through a microscope may be replaced by a velocity sensor, and the control circuit may in the future be integrated on a chip.

\section{Acknowledgement}

The authors would like to thank the National Science Council of the Republic of China, Taiwan, for financially supporting this research under Contract no. NSC94-2323-B-002-013.

\section{References}

[1] N.D. Cristescu, B.P. Conrad, R. Tran-Son-Tay, Int. J. Eng. Sci. 40 (2002) 605.

[2] C. Quilliet, B. Berge, Curr. Opin. Colloid Interf. Sci. 6 (2001) 34.

[3] M. Madou, Chapter 9, CRC, Boca Raton, FL, 1997.

[4] G.T.A. Kovacs, Chapter 9, McGraw-Hill, New York, 1998.

[5] C.-M. Ho, Proc. IEEE Int. Conf. MEMS, Interlaken, Switzerland, 2001, p. 375

[6] J. Lee, H. Moon, J. Fowler, T. Schoellhammer, C.J. Kim, Sens. Actuators A 95 (2002) 259.

[7] J. Lee, C.J. Kim, J. Microelectromech. Syst. 9 (2000) 171.

[8] H. Moon, S.K. Cho, R.L. Garrell, C.J. Kim, J. Appl. Phys. 92 (2002) 4080.

[9] S.K. Cho, H. Moon, C.J. Kim, J. Microelectromech. Syst. 12 (2003) 70.

[10] A.R. Wheeler, H. Moon, C.-J. Kim, Loo S J.A., R.L. Garrell, Anal. Chem. (2004) 4833.

[11] R. Feynman, R.B. Leighton, M.L. Sands, Feynman Lect. Phys. 2 (1964) 41.

[12] M.G. Pollack, A.D. Shenderov, R.B. Fair, Lab Chip 2 (2002) 96.

[13] V.K. Pamula, P.Y. Paik, J. Venkatraman, M.G. Pollack, R.B. Fair, Lab Chip 3 (2003) 28

[14] J. Lienemann, A. Greiner, J.G. Korvink, Nanotechnology 1 (2003) 94.

[15] E. Seyrat, R.A. Hayes, J. Appl. Phys. 90 (2001) 1383.

[16] F. Saeki, J. Baum, H. Moon, J. Yoon, C.J. Kim, R. Garrell, Polym. Mater.: Sci. Eng. 85 (2001) 12.

[17] C.H. Lin, G.B. Lee, B.W. Chang, G.L. Chang, J. Micromech. Microeng. 12 (2002) 590. 